\title{
COGNITIVE FRAILTY: RATIONAL AND DEFINITION FROM AN (I.A.N.A./I.A.G.G.) INTERNATIONAL CONSENSUS GROUP
}

\author{
E. KELAIDITI ${ }^{1}$, M. CESARI ${ }^{1,2,3}$, M. CANEVELLI ${ }^{1,4}$, G. ABELLAN VAN KAN $^{1,2}$, P.-J. OUSSET ${ }^{1}$, \\ S. GILLETTE-GUYONNET ${ }^{1,2}$, P. RITZ ${ }^{2,5}$, F. DUVEAU ${ }^{6}$, M.E. SOTO ${ }^{1,2}$, V. PROVENCHER ${ }^{7}$, \\ F. NOURHASHEMI ${ }^{1,2}$, A. SALVA ${ }^{8}$, P. ROBERT ${ }^{9}$, S. ANDRIEU ${ }^{1,2,3,10}$, Y. ROLLAND $^{1,2}$, J. TOUCHON ${ }^{11}$, \\ J.L. FITTEN ${ }^{12}$, B. VELLAS ${ }^{1,2,3}$
}

1. Gérontopôle, Centre Hospitalier Universitaire de Toulouse, France; 2. INSERM UMR 1027, Toulouse, France; 3. Université de Toulouse III Paul Sabatier, Toulouse, France; 4. Memory Clinic, Department of Neurology and Psychiatry, "Sapienza" University, Rome, Italy; 5. Pôle Cardiovasculaire et Métabolisme, Unité Transversale Nutrition Clinique, Hôpital Rangueil, Toulouse, France; 6. Médéos consulting, Garches, France; 7. Département de réadaptation, Université de Laval, Québec, Canada, and Centre de Recherche du CHU de Québec, Québec, Canada; 8. Institut Català de l'Envelliment, Universitat Autònoma de Barcelona, Barcelona, Spain; 9. Centre Mémoire de Ressources et de Recherche, CHU Nice, INSERM JE 2441 Neurobiologie et Psychopathologie, Nice, France; 10. Department of Public Heath, CHU de Toulouse; 11. Neurology Department, University Hospital of Montpellier, Montpellier, France; 12. David Geffen School of Medicine, UCLA, Los Angeles, USA and GLA VA Healthcare System, Sepulveda Campus, Los Angeles, USA. Correspondance to: Eirini Kelaiditi, Institut du Vieillissement, Gérontopôle, Université de Toulouse III-Paul Sabatier. 37 Allées Jules Guesde, 31000 Toulouse, France. Phone: +33 (0) 56114-5668; Email: kelaiditi.e@chu-toulouse.fr.

\begin{abstract}
The frailty syndrome has recently attracted attention of the scientific community and public health organizations as precursor and contributor of age-related conditions (particularly disability) in older persons. In parallel, dementia and cognitive disorders also represent major healthcare and social priorities. Although physical frailty and cognitive impairment have shown to be related in epidemiological studies, their pathophysiological mechanisms have been usually studied separately. An International Consensus Group on "Cognitive Frailty" was organized by the International Academy on Nutrition and Aging (I.A.N.A) and the International Association of Gerontology and Geriatrics (I.A.G.G) on April 16th, 2013 in Toulouse (France). The present report describes the results of the Consensus Group and provides the first definition of a "Cognitive Frailty" condition in older adults. Specific aim of this approach was to facilitate the design of future personalized preventive interventions in older persons. Finally, the Group discussed the use of multidomain interventions focused on the physical, nutritional, cognitive and psychological domains for improving the well-being and quality of life in the elderly. The consensus panel proposed the identification of the so-called "cognitive frailty" as an heterogeneous clinical manifestation characterized by the simultaneous presence of both physical frailty and cognitive impairment. In particular, the key factors defining such a condition include: 1) presence of physical frailty and cognitive impairment $(\mathrm{CDR}=0.5)$; and 2) exclusion of concurrent $\mathrm{AD}$ dementia or other dementias. Under different circumstances, cognitive frailty may represent a precursor of neurodegenerative processes. A potential for reversibility may also characterize this entity. A psychological component of the condition is evident and concurs at increasing the vulnerability of the individual to stressors.
\end{abstract}

Key words: Frailty, cognition, cognitive frailty, neurodegenerative disease, aging, elderly, disability, clinical markers, biological markers, neuroimaging, prevention.

\section{Introduction}

The aging of populations worldwide in the coming decades will lead to a marked increase of age-related diseases and disability with consequent profound personal, social and economic implications $(1,2)$. Given the difficulty in reversing aging's disabling cascades, it is important to act preventively with specifically tailored interventions against prodromal signs of disease and disability when these processes are still amenable to effective modification.

Recently, the frailty syndrome, which renders individuals more vulnerable to adverse health outcomes through generally subtle and progressive physical changes, has attracted attention in the medical and scientific communities as well as within the public health departments of numerous countries (3). In fact acting on frailty through effective interventions may change the aging trajectories of many individuals from the possible "pathological aging" pattern to the more personally and economically desirable "successful aging" (4).

Received June 14, 2013

Accepted for publication July 30, 2013
With regard to the brain, the role of brain aging, the development of brain frailty (i.e. a state of reduced neurophysiological reserves) and their relationship to both the late-life appearance of neurodegenerative and vascular diseases and to the appearance of physical frailty itself are not well understood. Recent demographic changes in industrialized populations have led to the emergence of the so-called "dementia epidemic". Available treatments for Alzheimer's disease (AD), for example, have only shown a moderate shortterm symptomatic effect without any disease-modifying efficacy. Moreover, several promising new drugs based mainly on the amyloid hypothesis have recently failed to alter established disease in phase 3 clinical trials (5). As a consequence, interest has developed in focusing on the preclinical stages of the dementia syndrome and on the identification of effective preventive strategies. Thus, similarly to disability prevention, there is an urgent need to act early, before neuronal damage becomes irreversible. 
At present, the concept of frailty has focused principally on the physical domain. However, some recent work has started considering cognition in the definition of frailty $(6,7)$. For example, a recent review paper by Clegg et al. included the pathophysiological pathway of brain aging among the causal factors of the frailty syndrome (8). Similarly, Buchman et al. have suggested that the accumulation of common brain pathological findings (e.g. Alzheimer's disease pathology, macroinfarcts, nigral neuronal loss) was independently associated with the rate of progression of physical frailty accounting for more than $8 \%$ of the variance unexplained by demographic variables alone in older participants from the Religious Orders Study and Memory and Aging Project (9). In view of the increased interest in the frailty syndrome and its relationship to the aging brain, and the continued goal of developing strategies to improve disability in the elderly, a workshop on Cognitive Frailty was conducted by an International Consensus Group on the 16th of April 2013 in Toulouse (France). The experts were chosen due to their expertise in different fields including frailty, Alzheimer's disease, public health, and geriatrics. The experts were selected by the Task Force chairs. A proposal paper was initially drafted and sent to the Consensus Group members prior to the meeting. The draft was presented and discussed during the meeting to reach consensus and formulate recommendations. The draft was revised after the meeting, resent to all co-authors for comments and a final version was commonly accepted by all members and the Task Force chairs. Discrepancies were discussed during the meeting and a final proposal was agreed.

Primary aim of the meeting was to discuss current issues related to the relationship existing between frailty and cognition. Specific objectives of the meeting were: i) to summarize the existing literature in order to identify the papers that have examined whether frailty is capable to predict cognitive outcomes, ii) to provide evidence showing links between frailty and cognition, iii) to discuss and propose a first definition on cognitive frailty, iv) to discuss and propose a list of screening tools and specific clinical and biological markers for identifying individuals at risk of physical disability and neurodegenerative disease, and v) discuss and propose potential preventive interventions about cognitive frailty.

\section{Physical frailty and cognitive impairment have been studied separately}

\section{Theoretical and operational definitions of frailty}

Frailty is a multidimensional geriatric syndrome characterized by increased vulnerability to stressors as a result of reduced capacity of different physiological systems $(10,11)$. It is also associated with increased risk of adverse healthrelated outcomes including falls, disability, hospitalizations and mortality (12-14). Although research on frailty has been ongoing for the last 15 years, there is still a lack of consensus about a unique operational definition (15-17). With progress in the identification of biomarkers and other advances in medicine, the definitions of diseases are constantly changing e.g. Alzheimer's disease, cancer, diabetes, and vascular diseases. This may partly explain the discrepancies in the prevalence rates of physical frailty, recently reported to vary widely ranging between $4.0-59.1 \%$ among older adults aged $\geq$ 65 years $(8,18)$.

To translate the theoretical concept of frailty into practice, several operational definitions of frailty have been proposed and are currently available in the literature. These emphasize principally physical frailty. For the purposes of this review, the main definitions are presented. The most commonly used definition of frailty was developed by Fried et al. as part of their work in the Cardiovascular Health Study (CHS) (11) and the Women's Health and Aging Studies (WHAS) [10]. Frailty is operationally defined as a clinical condition meeting 3 out of 5 criteria related to a physical phenotype including weak muscle strength, slow gait speed, unintentional weight loss, exhaustion and low physical activity (11). Although the physical phenotype of frailty has been validated and used in a number of studies $(14,15,19,20)$ many investigators have suggested a broader multidomain phenotype of frailty which is not limited strictly to the physical domain yet including diverse syndromes, diseases and disability states. Thus, Rockwood et al. operationalized the Frailty Index (FI) capturing the agerelated accumulation of clinical deficits, such as disability, diseases, physical and cognitive impairments, psychosocial risk factors and geriatric syndromes (such as falls, delirium and urinary incontinence) (21). The same group also developed the Clinical Frailty Scale based on 7 components ranging from 1 (robust health) to 7 (complete functional dependence on others) in order to provide a more clinical friendly instrument for the detection of frailty (7). Another operational definition of frailty was more recently developed and validated by Ensrud et al. in the Study of Osteoporotic Fractures (SOF) aiming to simplify the definition proposed by Fried et al. (22). This new screening instrument defines frailty as the presence of at least 2 out of 3 criteria including unintentional weight loss, inability to rise from a chair and exhaustion (23). Furthermore, Gobbens et al. have developed the Tilburg Frailty Indicator (TFI), a selfreported questionnaire to assess frailty (24). The TFI defines frailty including 3 domains (physical, psychological and social) and 15 single questions in these 3 domains. The score ranges from 0 to 15 , with higher score indicating higher risk of frailty.

Frailty has been predictive of poor clinical outcomes (e.g. falls, disability, hospitalization, mortality) (25-28), and has further been associated with biological abnormalities (e.g. biomarkers of inflammation) regardless of the definition used to assess frailty (29). These findings clearly suggest that frailty is influenced by a number of pathophysiological modifications involving the body's diverse physiological systems.

\section{Cognitive function in older persons}

Current research suggests that dementia represents the last phase of a progressive accumulation of pathological changes 
starting decades before the earliest clinical symptoms appear (30). In this regard, $\mathrm{AD}$, for example, is actually considered as a continuum of disease from preclinical asymptomatic to overt, symptomatic stages. Aiming at properly capturing and summarizing the course of the disease, several concepts/definitions have been proposed, both in clinical (31, 32) and research settings (33), to differentiate the different phases of the pathophysiological process (Table 1). These definitions have incorporated the recent scientific advances in the field, especially concerning biomarkers of the core features of AD (cerebrospinal liquid A $\beta 42$, Tau and phospho-Tau concentrations; amyloid deposition and glucose metabolism in positron emission tomography (PET); brain atrophy in magnetic resonance imaging (MRI)). Despite some slight discrepancies, there is a growing consensus in subdividing the course of $\mathrm{AD}$ and dementia into three subsequent stages: 1) a preclinical/asymptomatic stage, only revealed by biomarkers evidence; 2) a predementia phase, characterized by the impairment in memory or other cognitive domains not negatively affecting social and/or occupational functioning; and 3) a dementia phase, in which cognitive disturbances significantly interfere with the capacity for independent living. In fact, the interventions so far investigated to reduce dementia incidence have mainly failed, even if implemented in the Mild Cognitive Impairment (MCI)/prodromal stage (34) (probably because an extensive neuronal loss has already occurred).
Consequently, a strong effort is currently being developed to more clearly conceptualize a "pre-MCI" phase. In this context, a growing interest has emerged in "subjective cognitive complaints" (SCC), defined as self- or informant-reported cognitive disturbances occurring in the absence of an objective impairment of cognitive performance (35). Despite some conflicting results, SCC have been suggested as a potential risk factor for the eventual onset of cognitive impairment and dementia. Accordingly, dementia preventive trials (i.e. MAPT, GuidAge) are increasingly enrolling participants with subjective complaints as target populations $(36,37)$. Nevertheless, several methodological issues (i.e. broad heterogeneity of this entity, lack of standardized definitions and limited assessment tools) have so far limited the clinical and research adoption of the SCC concept. In conclusion, the definition and operationalization of a cognitive premorbid entity for dementia prevention is urgently needed.

\section{Physical and cognitive functional decline are linked}

\section{Clinical and subclinical factors shared between frailty and cognitive disorders}

Multiple subclinical and clinical conditions seem to underlie both the physical and cognitive age-related declines. For example, depression has been related to hippocampal atrophy and subsequent mild cognitive impairment (MCI) (38) as well

\section{Table 1}

Currently adopted definitions of the different dementia/AD stages

\begin{tabular}{|c|c|c|}
\hline Stage & Definition & Criteria \\
\hline \multicolumn{3}{|l|}{ Dementia phase } \\
\hline All cause dementia & $\begin{array}{l}\text { Cognitive and neuropsychiatric symptoms that: } 1 \text { ) interfere with normal functioning; } 2 \text { ) represent a } \\
\text { decline from previous levels of performing; } 3 \text { ) are not explained by delirium or other major psychiatric } \\
\text { disorders; 4) are detected by history-taking and objective cognitive assessment; 5) involve a } \\
\text { minimum of two cognitive/behavioral domains (memory, reasoning, visuospatial abilities, language, } \\
\text { personality) }\end{array}$ & NIA-AA \\
\hline Dementia due to AD & Dementia + evidence of AD biomarkers & NIA-AA \\
\hline AD dementia & Dementia + evidence of AD biomarkers & IWG \\
\hline \multicolumn{3}{|l|}{ Predementia phase } \\
\hline $\begin{array}{l}\text { Mild cognitive } \\
\text { impairment }(\mathrm{MCI})\end{array}$ & $\begin{array}{l}\text { 1) concerns reflecting a change in cognition reported by patient or informant or clinician; 2) objective } \\
\text { evidence of impairment in one or more cognitive domains; 3) preservation of independence in functional } \\
\text { abilities; 4) not demented. }\end{array}$ & NIA-AA \\
\hline Late MCI (LMCI) & $\begin{array}{l}\text { Concern on memory impairment and objective memory performance of } 1.5 \text { SD below the normative mean } \\
\text { in a standardized test }\end{array}$ & ADNI \\
\hline Early MCI (EMCI) & $\begin{array}{l}\text { Concern on memory impairment and objective memory performance between } 1.0 \mathrm{SD} \text { and } 1.5 \mathrm{SD} \\
\text { below the normative mean in a standardized test }\end{array}$ & ADNI \\
\hline $\mathrm{MCI}$ due to $\mathrm{AD}$ & $\mathrm{MCI}+$ evidence of $\mathrm{AD}$ biomarkers & NIA-AA \\
\hline Prodromal AD & $\begin{array}{l}\text { 1) impairment of cued recall in a memory task; 2) preservation of independence in functional abilities; } \\
\text { 3) evidence of AD biomarkers }\end{array}$ & IWG \\
\hline $\begin{array}{l}\text { Subjective cognitive } \\
\text { complaints/impairment } \\
\text { (SCC or SCI) }\end{array}$ & $\begin{array}{l}\text { 1) self- or informant-reported cognitive disturbances; } 2 \text { ) normal objective performance on } \\
\text { standardized cognitive testing (or performance less than } 1.0 \mathrm{SD} \text { ) }\end{array}$ & No criteria \\
\hline \multicolumn{3}{|l|}{ Preclinical phase } \\
\hline Preclinical stage of AD & No cognitive impairment on standard assessment + evidence of AD biomarkers & NIA-AA \\
\hline $\begin{array}{l}\text { Asymptomatic at-risk } \\
\text { stage of } \mathrm{AD}\end{array}$ & No cognitive impairment on standard assessment + evidence of AD biomarkers & IWG \\
\hline
\end{tabular}

NIA-AA: National Institute on Aging-Alzheimer's Association; IWG: International Working; Group for New Research Criteria for the Diagnosis of AD (81); ADNI: Alzheimer's; Disease Neuroimaging Initiative (82); SD: standard deviation 
as to the development or worsening of the physical frailty syndrome in older persons $(39,40)$. Similarly, cardiovascular risk factors (e.g. diabetes, dyslipidemia, hypertension, inflammation, hyperhomocysteinemia) may be responsible for cumulative neurological damage (41-43) and are positively associated with frailty (44-47). It has also been suggested that genetic mutations (e.g. APOE4) as well as environmental factors (e.g. low education, unhealthy dietary patterns, low physical and mental activity, smoking, high alcohol consumption) may negatively influence the aging brain $(43,48)$ rendering it more fragile and exposing it to the development of characteristic age-related diseases. Genetic (e.g. genes that are involved in apoptotic and transcription regulation pathways such as 5-methyltetrahydrofolate-homocysteine methyltranferase (MTR), Caspase 8 (CASP8), CREB-binding protein (CREBBP), lysine acetyltransferase 2B (KAT2B), and beta-transducin repeat containing (BTRC) loci) (49), and environmental factors (such as nutrition and physical activity) are also strongly related to frailty, both physical (50-52) and brain (53-55). Additional, pathophysiological mechanisms including oxidative damage and functional changes in the hippocampus and prefrontal cortex have been identified as important factors potentially mediating cognitive decline and potentially leading to dementia (56-58). Recently, Clegg et al. have suggested that structural and functional changes in the aging brain in combination with frailty may identify elderly people at particularly high risk of adverse outcomes (8).

\section{Physical frailty and the added value of cognition}

Cognitive impairment has increasingly been recognized as a component of frailty, contributing to its heterogeneity $(6,59)$. Interestingly, data from the French Three-City Study cohort, among older individuals aged 65-95 years, have incorporated cognitive impairment to the frailty phenotype and observed that cognitive impairment improved the predictive validity of the phenotype of frailty for adverse health outcomes over four years of follow-up (60).

A comprehensive review was performed in order to identify all relevant articles showing associations between the physical and cognitive domain of frailty (Figure 1). A Medline literature search of all articles published after 2001, when Fried et al. developed the first operational definition of frailty, was performed using the Medical Subject Heading (MeSH) terms "Human", "English", "Aged 65+ years" combined with the terms "Frailty" and "Cognition". Overall, 232 articles were retrieved. The identified abstracts were evaluated and for those articles that met the inclusion criteria the full articles were obtained in order to identify all relevant articles examining whether frailty is predictive of cognitive status. The consensus group had no interest at exploring the physical impairments due to cognitive decline because the underlying pathways for such relationship largely rely on neurodegenerative disorders and are already well know. Alternatively, the aim was to discuss current evidence potentially supporting a novel cognitive condition caused by physical frailty. Such a nosological entity is not yet described, but frequently identified in the assessment of older persons with physical frailty. A final selection of 9 articles was used for the purpose of this review shown in Table 2.

\section{Figure 1}

Flow chart of retrieved and selected articles showing associations between the physical and cognitive domain

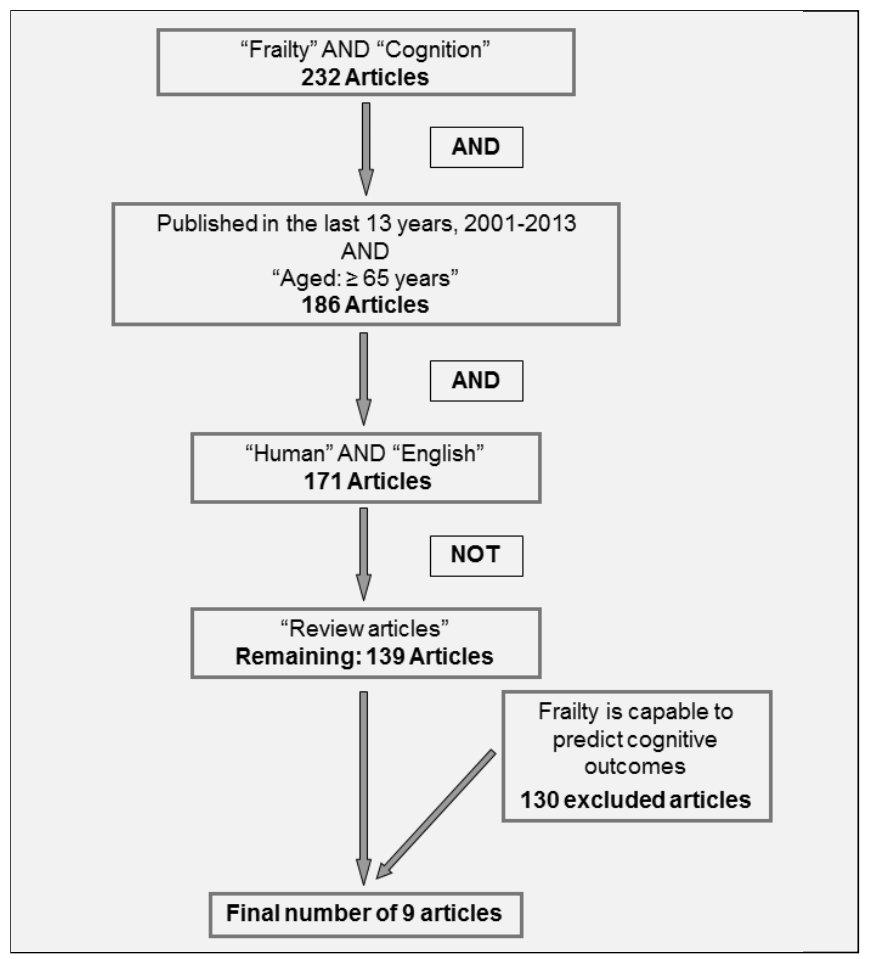

A number of longitudinal studies have indicated that frailty was associated with low cognitive performance over time in older individuals with and without dementia (61-67) and crosssectional analyses, although limited, have also reported associations between physical frailty and cognitive decline (68, 69). In the most recent prospective study, Gray et al. observed that in a large population of older adults aged $\geq 65$ years, frailty was associated with a 2.57 -fold increased risk for non-AD dementia, although they did not observe any associations between frailty and all-cause dementia or AD (66). Findings from this study are contradictory and suggest that the frailty phenotype may not be predictive for dementia. Moreover, there was a considerable variability in participants' age and on the assessment of frailty, so it was difficult to draw direct comparisons. In summary, to date, studies on frailty and cognition are associational in nature and do not demonstrate causal links. Further research is needed to elucidate the links between physical frailty and cognitive performance.

The inclusion of cognition in the operational definition of frailty may also be important for meeting the multiple 
The Journal of Nutrition, Health \& Aging(C)

Volume 17, Number 9, 2013

COGNITIVE FRAILTY: RATIONAL AND DEFINITION FROM AN (I.A.N.A.II.A.G.G.)

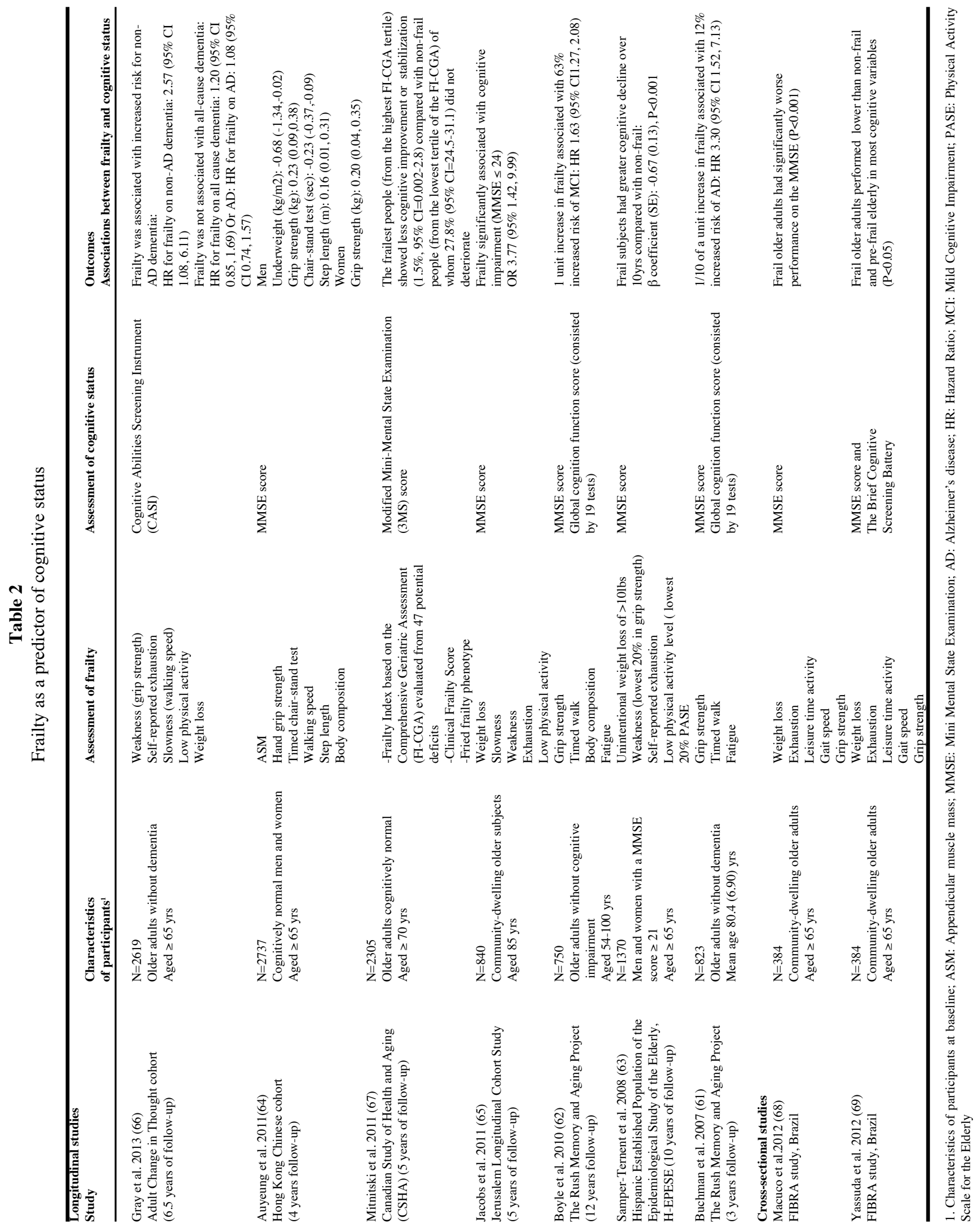


pathophysiological mechanisms shared by both frailty and neurodegenerative disorders (70).

\section{Physical and cognitive decline in frailty}

An example of the close relationship between physical and cognitive decline in older persons can be directly observed in the clinical practice. Recently, the Gérontopôle of the Centre Hospitalier Universitaire de Toulouse (France) in collaboration with the University Department of General Medicine of Toulouse (DUMG) and the regional health authority (Agence Regionale de Santé of the Midi Pyrénées region, France) has developed an innovative Platform for the Evaluation of Frailty and the Prevention of Disability, thus integrating frailty into clinical practice. After the first 6 months of operation and evaluation of $(n=160)$ older adults, data show that more than half of the assessed frail individuals $(52.9 \%)$ presented a Clinical Dementia Rating score (CDR) of 0.5 revealing an objective cognitive impairment (71). In fact, whereas some of these patients are likely to present an early phase of a neurodegenerative condition (not yet diagnosed), or show a non-dementia more accelerated decline, it is possible that many others may not experience a further cognitive decline (Figure 2). The need for distinguishing different risk profiles (in terms of future health-related events) within a unique theoretical definition of frailty is crucial in order to adopt the adequate countermeasures and allow for the development of specific personalized intervention programs. In this context, it is noteworthy that physical impairment is often responsible for increasing sedentary behavior and social isolation in older persons. These two factors do not only concur at determining a vicious cycle detrimental for the physical domain of the individual, but may also explain a cognitive decline caused by factors independent of a neurodegenerative condition that should be treated differently.

\section{Panel discussion on the definition of Cognitive Frailty in older adults}

Older non-demented persons can be operationally categorized into four groups according to their physical and cognitive status. These groups include:

(i) Robust older individuals (i.e., no evidence of physical frailty) without cognitive problems (i.e., normal brain aging);

(ii) Physically frail older adults with normal cognitive functioning (as indicated by a Clinical Dementia Rating [CDR] equal to 0 ), including individuals with subjective memory complaints;

(iii) Older adults with no physical frailty but already exhibiting a cognitive impairment $(\mathrm{CDR}=0.5)$;

(iv) Physically frail older adults with cognitive impairment $(\mathrm{CDR}=0.5)$.
The consensus panel was particularly interested at discussing about this latter group (iv), in order to evaluate whether cognitive impairment may be linked to physical impairment rather than neurodegenerative disorders, consequently making cognition a relevant part of the frailty syndrome.

\section{Figure 2}

Different trajectories of cognitive function according to specific conditions

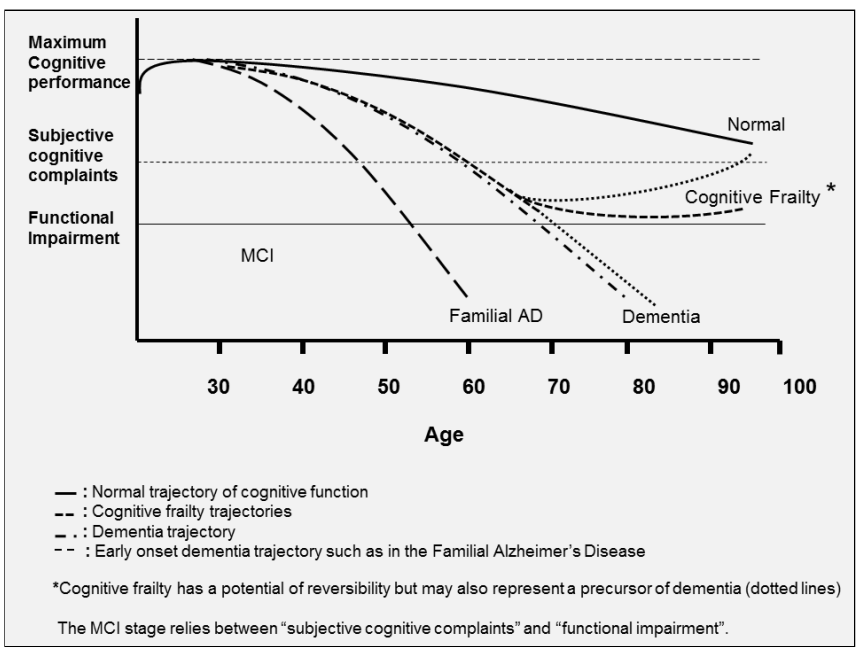

Proposed definition of cognitive frailty

After evaluating the current literature, the consensus panel proposed the identification of the so-called "cognitive frailty" as an heterogeneous clinical manifestation characterized by the simultaneous presence of both physical frailty and cognitive impairment. In particular, the key factors defining such a condition include:

- Presence of physical frailty and cognitive impairment $(\mathrm{CDR}=0.5)$;

- Exclusion of concurrent AD dementia or other dementias.

The two defining criteria imply that cognitive frailty is characterized by reduced cognitive reserve, but is different from the physiological brain aging. At the same time, it is noteworthy that, under different circumstances, cognitive frailty may also represent a precursor of neurodegenerative processes. A potential for reversibility may also characterize cognitive frailty. A psychological component of the condition is evident and concurs at increasing the vulnerability of the individual to stressors.

The proposed definition addresses a current gap in the existing literature, as it particularly allows the conceptualization of a cognitive impairment due to the physical domain of the individual and not to the presence of a concomitant neurological disease (Figure 2). In other words, this represents the first attempt to identify a clinical entity including both physical and cognitive dimensions. In fact, 
dementia and disability are complex conditions and should be not attributed to a single domain. The identification of cognitive frailty in older persons may support the design of preventive and/or rehabilitative interventions, for which specific clinical settings might be amenable in the future. Moreover, the paper mentioned Alzheimer disease more than other forms of dementia since this is the most common type of neurodegenerative disorder. However, it is not in the aim of the consensus group to study neurodegenerative disorders but a condition characterized by cognitive impairment due to physical conditions. As a further clarification, the frailty syndrome (although phenotypically driven by the physical domain) is a systemic condition. By using the term "cognitive frailty", the consensus wanted to identify a condition of cognitive impairment caused by physical conditions.

\section{Development of adequate screening tools and specific clinical and biological markers}

In order to design effective interventions for cognitive frailty, effective screening and diagnostic tools exploring and identifying multiple domains/causes of frailty including cognitive and psychological status need to be developed. In addition to improved screening instruments, biomarkers for improving the determination of the risk for physical impairment rather than neurodegenerative disease (and vice versa) need to be identified. This will provide us with the opportunity to better detect the possible future health trajectories that a frail person with cognitive impairment will follow. Consequently, this differentiation will allow the design of better personalized preventive or therapeutic interventions.

Possible biomarkers, clinical markers and imaging techniques that could be used to characterize and eventually to predict distinct age trajectories were suggested by the expert panel and are presented in Table 3. This list does not aim to be complete, accurate, or exhaustive, however it is only indicative and provides a rough description of the usefulness of different parameters/techniques in the assessment of physical and cognitive outcomes.

To identify cognitive frailty, the panel suggested that all the frail subjects should perform a comprehensive cognitive assessment exploring memory performance as well as other cognitive functions (i.e. executive functions). Several cognitive tests and instruments were suggested such as speed of processing test, the Montreal Cognitive Assessment test (MoCA), the Mini Mental State Examination (MMSE) and the Alzheimer's disease Assessment Scale-cognitive subscale (ADAS-Cog). After cognitive assessment, a complete physical assessment should be performed, which would help to identify which conditions are associated to physical impairment. Physical assessment tools, such as gait speed, hand grip strength and weight loss were also recommended (72). In addition, nutritional assessment would help to identify any dietary deficits, and a brief psychological assessment including tools such as the Geriatric depression scale (GDS) will help to identify any causes of reduction of energy level. Before proceeding in more sophisticated diagnostic procedures including imaging techniques, such as computed tomography (CT) and magnetic resonance imaging (MRI), correction for reversible conditions was suggested. Other diagnostic techniques can be considered for research purposes. Actigraphy (73) and accelerometer-based measures (74) may be of interest for measuring both physical activity and behavioral patterns. Dual energy X-ray absorptiometry scans (DEXA), may provide supplementary information about body composition. Functional magnetic resonance imaging (f-MRI), Diffusion tensor imaging (DTI), tractography, and cognitive evoked potentials could be particularly useful cognitive research tools.

\section{Table 3}

Possible biological, clinical, and imaging markers for identifying risk of physical disability and neurodegenerative disease

\begin{tabular}{lcc}
\hline & $\begin{array}{c}\text { Physical } \\
\text { disability }\end{array}$ & $\begin{array}{c}\text { Neurodegenerative } \\
\text { disease }\end{array}$ \\
\hline Biomarkers & & \\
Inflammatory markers & +++ & +++ \\
(e.g. CRP, IL-6) & & \\
Beta-amyloid protein (A $\beta$ ) & - & ++ \\
APOEE4 genotype & - & ++ \\
Anemia & ++ & ++ \\
Serum Albumin & ++ & ++ \\
Cholesterol & ++ & ++ \\
Vitamin D status & ++ & ++ \\
Clinical markers & & \\
MMSE & ++ & +++ \\
Executive tests & + & +++ \\
ADAS-Cog & - & +++ \\
CDR & ++ & +++ \\
MoCA & ++ & +++ \\
Gait speed & +++ & ++ \\
Hand grip strength & +++ & ++ \\
Weight loss & +++ & ++ \\
Psychological marker: GDS & ++ & +++ \\
Actigraphy & +++ & +++ \\
Imaging & & ++ \\
Dual energy X-ray absorptiometry & ++ & \\
scans (DEXA) & & ++ \\
Cerebral Computed tomography (CT scans) & + & ++ \\
Cerebral Magnetic resonance imaging (MRI) & + & +++ \\
Functional magnetic resonance imaging & + & +++ \\
(FMRI) & & +++ \\
Diffusion tensor imaging (DTI) & + & ++ \\
Tractography & + & ++ \\
Electrophysiological methods & + & ++ \\
Cognitive evoked potentials & + & ++ \\
\hline & & ++ \\
& ++ & ++ \\
\hline
\end{tabular}

: not recommended,+: may be of use, ++ : suitable for use, +++ : recommended for use CRP: C-reactive protein; IL-6: Interleukin-6; APOE 4 : Apolipoprotein E $\varepsilon 4$ allele; MMSE: Mini Mental State Examination; ADAS-Cog: Alzheimer's disease Assessment Scale-cognitive subscale; CDR: Clinical Dementia Rating; MoCA: Montreal Cognitive Assessment; GDS: Geriatric Depression Scale

In parallel, usual physical frailty markers (such as weight loss and gait speed) should be assessed in persons exhibiting a cognitive decline, even if at early stages.

Some markers may be able to well capture both the risk of future physical and cognitive declines, such as inflammatory 
biomarkers [e.g. C-reactive protein (CRP) and Interleukin-6 (IL-6)] $(28,75,76-78)$. However, biomarkers predictive of both types of decline may not be particularly useful in differentiating whether a person is at higher risk of a future physical rather than a cognitive decline (and vice versa). Other markers may better serve at estimating the specific risk for one single domain. For example, the beta-amyloid protein $(\mathrm{A} \beta)$ and the apolipoprotein APOE\&4 genotype are closely related to the development of dementia and Alzheimer's disease (79).

\section{Potential preventive interventions}

Including cognition in the definition of frailty and exploring the different health trajectories that a frail person with subjective cognitive impairment will follow is emerging. Research in this direction will further inform public health policies to implement evidence-based research findings to the development of prevention plans and clinical trials. Initially, a list of preventive interventions may be considered. These may include promotion of physical activity, cognitive stimulation and training, healthy dietary habits (e.g. the Mediterranean diet), smoking cessation, promotion of emotional resilience, active and socially integrated lifestyles, optimal daily sleep, maintenance of optimal body weight, and metabolic control (including control of dyslipidemia, diabetes and blood pressure) (80). As a further step the causes of frailty need to be identified to enable the implementation of multidomain interventions based on evidence-based research and depending on personalized needs. Evaluation of pharmacological therapy and drug use are also recommended. Multidomain interventions might prove useful if focused on the physical, nutritional, cognitive and psychological domains in order to improve the well-being and quality of life in the elderly. The promotion of physical exercise, correction of nutrient deficiencies, potential nutrient supplementation, and implementation of cognitive training (including a psychological perspective at the same time with other interventions) may be considered to more comprehensively improve well-being and the quality of daily life in older persons. These strategies may result ineffective if focused on single components and, thus, failing to capture the complexity of the phenomenon.

\section{Concluding remarks}

Cognitive frailty may represent a novel concept to consider in the complex and heterogeneous scenario of frailty in older persons. It is hoped that this initial discussion between experts from clinical, research and industry fields may provide a stimulus for development of new research in the field. The consensus group proposed the hypothesis of a possible new condition. There was no intention of operationalizing it, because available data are still preliminary and far to be conclusive. Aim of the consensus was indeed to stimulate research in the field. A continued dialogue on this topic would benefit investigators with an interest in cognitive frailty to develop new studies with the aim to better understand this condition. It is also important that future research in the field may inform public health policies for the implementation of prevention and treatment programs.

Conflicts of interest: Matteo Cesari has been consultant for Nutricia, Sanofi and Nestlé, and has a research project funded by Pfizer. The other authors had no conflicts of interest to disclose related to this manuscript. This work was supported by an unrestricted educational grant from Nestlé Health Science (NHSc). NHSc had no role in the choice of members of the Consensus Group, but had the right to have an observer member at the meeting. Members of the Consensus Group received no salary in the preparation of this manuscript.

\section{References}

1. Beard J, Biggs S, Bloom D, et al. (2011) Global Population Ageing: Peril or Promise, Geneva: World Economic Forum, 2011.

2. Gonyea J (2005) The economic well-being of older Americans and the persistent divide. Public Health \& Aging Report, 15 p. 1-11.

3. Morley JE (2011) Frailty: diagnosis and management. J Nutr Health Aging, 15 (8): p. $667-$ 70 .

4. Cesari M, Vellas B and Gambassi G (2013) The stress of aging. Exp Gerontol, 48 (4): p. 451-6.

5. Green R, Schneider L, Amato D, et al. (2009) Effect of tarenflurbil on cognitive decline and activities of daily living in patients with mild alzheimer disease: A randomized controlled trial. JAMA, 302 (23): p. 2557-2564.

6. Bergman H, Ferrucci L, Guralnik J, et al. (2007) Frailty: an emerging research and clinical paradigm--issues and controversies. J Gerontol A Biol Sci Med Sci, 62 (7): p. 731-7.

7. Rockwood K, Song X, MacKnight C, et al. (2005) A global clinical measure of fitness and frailty in elderly people. CMAJ, 173 (5): p. 489-95.

8. Clegg A, Young J, Iliffe S, et al. (2013) Frailty in elderly people. Lancet, 381 (9868): p. 752-62.

9. Buchman A, Yu L, Wilson R, et al. (2013) Association of brain pathology with the progression of frailty in older adults. Neurology, 80 p. 1-7.

10. Bandeen-Roche K, Xue QL, Ferrucci L, et al. (2006) Phenotype of frailty: characterization in the women's health and aging studies. J Gerontol A Biol Sci Med Sci, 61 (3): p. 262-6.

11. Fried LP, Tangen CM, Walston J, et al. (2001) Frailty in older adults: evidence for a phenotype. J Gerontol A Biol Sci Med Sci, 56 (3): p. M146-56.

12. Ensrud KE, Blackwell TL, Redline S, et al. (2009) Sleep disturbances and frailty status in older community-dwelling men. J Am Geriatr Soc, 57 (11): p. 2085-93.

13. Gill T, Gahbauer E, Allore H, et al. (2006) Transitions between frailty states among community-living older persons. Arch Intern Med, 166 (4): p. 418-23.

14. Graham JE, Snih SA, Berges IM, et al. (2009) Frailty and 10-year mortality in communityliving Mexican American older adults. Gerontology, 55 (6): p. 644-51.

15. Abellan van Kan G, Rolland Y, Bergman H, et al. (2008) The I.A.N.A Task Force on frailty assessment of older people in clinical practice. J Nutr Health Aging, 12 (1): p. 2937.

16. Abellan van Kan G, Rolland Y, Houles M, et al. (2010) The assessment of frailty in older people. Clin Geriatr Med, p. 275-286.

17. Rodríguez-Mañas L, Féart C, Mann G, et al. (2013) Searching for an Operational Definition of Frailty: A Delphi Method Based Consensus Statement. The Frailty Operative Definition-Consensus Conference Project. The Journals of Gerontology Series A: Biological Sciences and Medical Sciences, 68 (1): p. $62-67$.

18. Collard RM, Boter H, Schoevers RA, et al. (2012) Prevalence of frailty in communitydwelling older persons: a systematic review. J Am Geriatr Soc, 60 (8): p. 1487-92.

19. Fried LP, Ferrucci L, Darer J, et al. (2004) Untangling the concepts of disability, frailty, and comorbidity: implications for improved targeting and care. J Gerontol A Biol Sci Med Sci, 59 (3): p. 255-63.

20. Rothman MD, Leo-Summers L and Gill TM (2008) Prognostic significance of potential frailty criteria. J Am Geriatr Soc, 56 (12): p. 2211-116.

21. Rockwood K, Stadnyk K, MacKnight C, et al. (1999) A brief clinical instrument to classify frailty in elderly people. Lancet, 353 (9148): p. 205-6.

22. Ensrud KE, Ewing SK, Taylor BC, et al. (2008) Comparison of 2 frailty indexes for prediction of falls, disability, fractures, and death in older women. Arch Intern Med, 168 (4): p. 382-9.

23. Yesavage JA, Brink TL, Rose TL, et al. (1982) Development and validation of a geriatric depression screening scale: a preliminary report. J Psychiatr Res, 17 (1): p. 37-49.

24. Gobbens RJ, van Assen MA, Luijkx KG, et al. (2010) Determinants of frailty. J Am Med Dir Assoc, 11 (5): p. 356-64.

25. Gobbens RJJ, van Assen MALM, Luijkx KG, et al. (2012) The Predictive Validity of the Tilburg Frailty Indicator: Disability, Health Care Utilization, and Quality of Life in a Population at Risk. The Gerontologist, 52 (5): p. 619-631.

26. Shim EY, Ma SH, Hong SH, et al. (2011) Correlation between Frailty Level and Adverse Health-related Outcomes of Community-Dwelling Elderly, One Year Retrospective Study. Korean J Fam Med, 32 (4): p. 249-56. 


\section{COGNITIVE FRAILTY: RATIONAL AND DEFINITION FROM AN (I.A.N.A./I.A.G.G.)}

27. Theou O, Rockwood M, Mitniski A, et al. (2012) Disability and co-morbidity in relation to frailty: How much do they overlap? Arch Gerontol Geriatr, 55 (2): p. e1-e8.

28. Walston J, McBurnie MA, Newman A, et al. (2002) Frailty and activation of the inflammation and coagulation systems with and without clinical comorbidities: results from the Cardiovascular Health Study. Arch Intern Med, 162 (20): p. 2333-41.

29. Hubbard RE, O’Mahony MS, Savva GM, et al. (2009) Inflammation and frailty measures in older people. Journal of Cellular and Molecular Medicine, 13 (9b): p. 3103-3109.

30. Jack CR, Jr., Knopman DS, Jagust WJ, et al. (2013) Tracking pathophysiological processes in Alzheimer's disease: an updated hypothetical model of dynamic biomarkers. Lancet Neurol, 12 (2): p. 207-16.

31. Albert M, DeKosky S, Dickson D, et al. (2011) The diagnosis of mild cognitive impairment due to Alzheimer's disease: Recommendations from the National Institute on Aging-Alzheimer's Association workgroups on diagnostic guidelines for Alzheimer's disease. Alzheimer's \& Dementia, 7 (3): p. 270-279.

32. McKhann G, Knopman D, Chertkow H, et al. (2011) The diagnosis of dementia due to Alzheimer's disease: Recommendations from the National Institute on Aging-Alzheimer's Association workgroups on diagnostic guidelines for Alzheimer's disease. Alzheimer's \& Dementia, 7 p. 263-269.

33. Sperling R, Aisen P, Beckett L, et al. (2011) Toward defining the preclinical stages of Alzheimer's disease: Recommendations from the National Institute on Aging-Alzheimer's Association workgroups on diagnostic guidelines for Alzheimer's disease. Alzheimer's \& Dementia, 7 p. 280-292.

34. Aisen P (2008) Treatment for MCI: is the evidence sufficient? Neurology, 70 (22): p 2020-1.

35. Abdulrab K and Heun R (2008) Subjective memory impairement. A review of it definitions indicates the need for a comprehensive set of standardised and validated criteria. European Phychiatry, 23 (5): p. 321-330.

36. Gillette-Guyonnet S, Andrieu S, Dantoine T, et al. (2009) Commentary on "A roadmap for the prevention of dementia II. Leon Thal Symposium 2008." The Multidomain Alzheimer Preventive Trial (MAPT): A new approach to the prevention of Alzheimer's disease. Alzheimer's \& Dementia, 5 (2): p. 114-121.

37. Vellas B, Coley N, Ousset P-J, et al. (2012) Long-term use of standardised ginkgo biloba extract for the prevention of Alzheimer's disease (GuidAge): a randomised placebocontrolled trial. The Lancet Neurology, 11 (10): p. 851-859.

38. Panza F, Frisardi V, Capurso C, et al. (2010) Late-life depression, mild cognitive impairment, and dementia: possible continuum? Am J Geriatr Psychiatry, 18 (2): p. 98116

39. Mezuk B, Edwards L, Lohman M, et al. (2012) Depression and frailty in later life: a synthetic review. International Journal of Geriatric Psychiatry, 27 (9): p. 879-892.

40. Lohman MC and Mezuk B (2013) Frailty and Depression: Comorbidity in the Context of Imperfect Measurement. Journal of the American Geriatrics Society, 61 (3): p. 474-474.

41. Jefferson AL, Himali JJ, Au R, et al. (2011) Relation of left ventricular ejection fraction to cognitive aging (from the Framingham Heart Study). Am J Cardiol, 108 (9): p. 1346-51.

42. Jefferson AL, Himali JJ, Beiser AS, et al. (2010) Cardiac index is associated with brain aging: the Framingham Heart Study. Circulation, 122 (7): p. 690-7.

43. Kamat SM, Kamat AS and Grossberg GT (2010) Dementia risk prediction: are we there yet? Clin Geriatr Med, 26 (1): p. 113-23.

44. Collerton J, Martin-Ruiz C, Davies K, et al. (2012) Frailty and the role of inflammation, immunosenescence and cellular ageing in the very old: cross-sectional findings from the Newcastle 85+ Study. Mech Ageing Dev, 133 (6): p. 456-66.

45. Phan HM, Alpert JS and Fain M (2008) Frailty, inflammation, and cardiovascular disease: evidence of a connection. Am J Geriatr Cardiol, 17 (2): p. 101-7.

46. Kalyani RR, Varadhan R, Weiss CO, et al. (2012) Frailty Status and Altered GlucoseInsulin Dynamics. The Journals of Gerontology Series A: Biological Sciences and Medical Sciences, 67 (12): p. 1300-1306

47. Lu FP, Lin KP and Kuo HK (2009) Diabetes and the risk of multi-system aging phenotypes: a systematic review and meta-analysis. PLoS One, 4 (1): p. e4144.

48. Morley JE (2010) Nutrition and the brain. Clin Geriatr Med, 26 (1): p. 89-98.

49. Ho Y-Y, Matteini AM, Beamer B, et al. (2011) Exploring Biologically Relevant Pathways in Frailty. The Journals of Gerontology Series A: Biological Sciences and Medical Sciences, 66A (9): p. 975-979.

50. Dato S, Montesanto A, Lagani V, et al. (2012) Frailty phenotypes in the elderly based on cluster analysis: a longitudinal study of two Danish cohorts. Evidence for a genetic influence on frailty. AGE, 34 (3): p. 571-582.

51. Kaiser M, Bandinelli S and Lunenfeld B (2010) Frailty and the role of nutrition in older people. A review of the current literature. Acta Biomed, 81 Suppl 1 p. 37-45.

52. Smit E, Winters-Stone KM, Loprinzi PD, et al. (2012) Lower nutritional status and higher food insufficiency in frail older US adults. British Journal of Nutrition, FirstView p. 1-7.

53. Gates N, Fiatarone Singh MA, Sachdev PS, et al. (2013) The Effect of Exercise Training on Cognitive Function in Older Adults with Mild Cognitive Impairment: A Meta-analysis of Randomized Controlled Trials. The American Journal of Geriatric Psychiatry, (0): In press.

54. Mi W, van Wijk N, Cansev M, et al. (2013) Nutritional approaches in the risk reduction and management of Alzheimer's disease. Nutrition, 29(9): p. 1080-9

55. Presse N, Belleville S, Gaudreau P, et al. (2013) Vitamin K status and cognitive function in healthy older adults. Neurobiology of Aging, (0): In press.

56. Boveris A and Navarro A (2008) Brain mitochondrial dysfunction in aging. IUBMB Life,
60 (5): p. 308-14

57. Bishop NA, Lu T and Yankner BA (2010) Neural mechanisms of ageing and cognitive decline. Nature, 464 (7288): p. 529-35.

58. Panegyres PK (2004) The contribution of the study of neurodegenerative disorders to the understanding of human memory. QJM, 97 (9): p. 555-67.

59. Rockwood K (2005) Frailty and its definition: a worthy challenge. J Am Geriatr Soc, 53 (6): p. 1069-70.

60. Ávila-Funes JA, Amieva H, Barberger-Gateau P, et al. (2009) Cognitive Impairment Improves the Predictive Validity of the Phenotype of Frailty for Adverse Health Outcomes: The Three-City Study. Journal of the American Geriatrics Society, 57 (3): p. 453-461.

61. Buchman AS, Boyle PA, Wilson RS, et al. (2007) Frailty is Associated With Inciden Alzheimer's Disease and Cognitive Decline in the Elderly. Psychosomatic Medicine, 69 (5): p. 483-489.

62. Boyle PA, Buchman AS, Wilson RS, et al. (2010) Physical Frailty Is Associated with Incident Mild Cognitive Impairment in Community-Based Older Persons. Journal of the American Geriatrics Society, 58 (2): p. 248-255.

63. Samper-Ternent R, Al Snih S, Raji MA, et al. (2008) Relationship between frailty and cognitive decline in older Mexican Americans. J Am Geriatr Soc, 56 (10): p. 1845-52.

64. Auyeung TW, Lee JS, Kwok T, et al. (2011) Physical frailty predicts future cognitive decline - a four-year prospective study in 2737 cognitively normal older adults. J Nutr Health Aging, 15 (8): p. 690-4.

65. Jacobs J, Cohen A, Ein-Mor E, et al. (2011) Frailty, cognitive impairment and mortality among the oldest old. The journal of nutrition, health \& aging, 15 (8): p. 678-682.

66. Gray SL, Anderson ML, Hubbard RA, et al. (2013) Frailty and Incident Dementia. J Gerontol A Biol Sci Med Sci, 68(9): 1083-90

67. Mitnitski A, Fallah N, Rockwood MRH, et al. (2011) Transitions in cognitive status in relation to frailty in older adults: A Comparison of three frailty measures. The journal of nutrition, health \& aging, 15 (10): p. 863-867.

68. Macuco CRM, Batistoni SST, Lopes A, et al. (2012) Mini-Mental State Examination performance in frail, pre-frail, and non-frail community dwelling older adults in Ermelino Matarazzo, São Paulo, Brazil. International Psychogeriatrics, 24 (11): p. 1725-1731.

69. Yassuda M, Lopes A, Cachioni M, et al. (2012) Frailty criteria and cognitive performance are related: Data from the Fibra study in Ermelino Matarazzo, Sao Paulo, Brazil. The journal of nutrition, health \& aging, 16 (1): p. 55-61.

70. Houles M, Canevelli M, Abellan van Kan G, et al. (2012) Frailty and Cognition. The Journal of Frailty \& Aging, 1 (2): p. 56-63.

71. Subra J, Gillette-Guyonnet S, Cesari M, et al. (2012) The integration of frailty into clinical practice: Preliminary results from the Gérontopôle. The journal of nutrition, health \& aging, 16 (8): p. 714-720

72. Abellan van Kan G, Rolland Y, Andrieu S, et al. (2009) Gait speed at usual pace as apredictor of adverse outcomes in community-dwelling plder people. An International Academy on Nutrition and Aging (IANA) Task Force. . The Journal of Nutrition \& Aging, 13 (10): p. 881-889.

73. David R, Mulin E, Friedman L, et al. (2012) Decreased daytime motor activity associated with apathy in Alzheimer disease: an actigraphic study. Am J Geriatr Psychiatry, 20 (9): p. 806-14.

74. Semanik P, Lee J, Manheim L, et al. (2011) Relationship between accelerometer-based measures of physical activity and the Yale Physical Activity Survey in adults with arthritis. Arthritis Care \& Research, 63 (12): p. 1766-1772.

75. Leng S, Chaves P, Koenig K, et al. (2002) Serum interleukin-6 and hemoglobin as physiological correlates in the geriatric syndrome of frailty: a pilot study. J Am Geriatr Soc, 50 (7): p. 1268-71.

76. Wilson CJ, Finch CE and Cohen HJ (2002) Cytokines and cognition--the case for a headto-toe inflammatory paradigm. J Am Geriatr Soc, 50 (12): p. 2041-56.

77. Engelhart MJ, Geerlings MI, Meijer J, et al. (2004) Inflammatory proteins in plasma and the risk of dementia: the rotterdam study. Arch Neurol, 61 (5): p. 668-72.

78. Ravaglia G, Forti P, Maioli F, et al. (2005) Serum C-reactive protein and cognitive function in healthy elderly Italian community dwellers. J Gerontol A Biol Sci Med Sci, 60 (8): p. 1017-21.

79. Albert MS, DeKosky ST, Dickson D, et al. (2011) The diagnosis of mild cognitive impairment due to Alzheimer's disease: recommendations from the National Institute on Aging-Alzheimer's Association workgroups on diagnostic guidelines for Alzheimer's disease. Alzheimers Dement, 7 (3): p. 270-9.

80. Desai AK, Grossberg GT and Chibnall JT (2010) Healthy brain aging: a road map. Clin Geriatr Med, 26 (1): p. 1-16.

81. Dubois B, Feldman HH, Jacova C, et al. (2010) Revising the definition of Alzheimer's disease: a new lexicon. Lancet Neurol, 9 (11): p. 1118-27.

82. Aisen PS, Petersen RC, Donohue MC, et al. (2010) Clinical Core of the Alzheimer's Disease Neuroimaging Initiative: progress and plans. Alzheimer's \& Dementia, 6 (3): p. $239-46$ 